\title{
Fabrication of silicon-based optical components for an ultraclean accelerator mass spectrometry negative ion source
}

\author{
J. F. Kirchhoff, D. K. Marble, D. L. Weathers, F. D. McDaniel, and S. Matteson \\ Ion Beam Modification and Analysis Laboratory, Department of Physics, University of North Texas, Denton, \\ Texas 76203 \\ J. M. Anthony, R. L. Beavers, and T. J. Bennett \\ Central Research Laboratory, Texas Instruments, Inc., Dallas, Texas 75265
}

(Received 26 August 1993; accepted for publication 20 January 1994)

\begin{abstract}
An ultraclean accelerator mass spectrometry negative ion source for semiconductor material mass analysis has been built and is in operation at the University of North Texas' Ion Beam Modification and Analysis Laboratory (IBMAL). The source is unique in that the active surfaces and apertures of the optical components in the ion source have been fabricated from high-purity single crystal silicon. This prevents both the ${ }^{133} \mathrm{Cs}^{+}$beam incident on the semiconductor samples and the negative ions from the sample surfaces from "seeing" and sputtering any metal surfaces (mostly stainless steel) in the beamline. The $\mathrm{Cs}^{+}$beam can be rastered across the sample surface and the impact energy is adjustable to control depth-profiling rates. An ultraclean ion source of this type is necessary to prevent the injection of $\mathrm{Fe}$ and other beamline elements onto the sample or into the tandem accelerator, which is equivalent to putting an impurity signal into the mass analysis of the semiconductor sample. Suppression of these elements increases the sensitivity of the analysis to one part in $10^{12}$ for many masses. The fabrication and alignment of the optical components-einzel lenses, steerers, raster/scanners, and Faraday cups-in the negative ion source will be presented. Also, simulation trajectories are presented to show (1) the interaction of the incident $\mathrm{Cs}^{+}$ion beam, the semiconductor sample, and holder (biased up to $-30 \mathrm{kV}$ ), the extracted beam in the sample chamber, and associated potential field lines; and (2) the potential field lines of the octupole steerers.
\end{abstract}

\section{INTRODUCTION}

The University of North Texas' Ion Beam Modification and Analysis Laboratory (UNT-IBMAL) ${ }^{1}$ has developed an ultraclean, negative ion source for semiconductor impurity analysis via accelerator mass spectrometry (AMS). AMS has been used extensively for radioisotope dating, but has only recently been applied to materials analysis. ${ }^{2-6}$ The attractiveness of AMS stems from the ability to remove molecular ion interferences by Coulomb exploding the molecules as they pass through the terminal of a tandem accelerator. ${ }^{7,8}$

In the terminal of the UNT tandem accelerator, nitrogen gas is confined to a small interaction chamber at which the incident negative ions (molecular and atomic) interact with the nitrogen atoms. The subsequent collisions result in electrons being stripped from the negative ions and, for high enough charge states, a dissociation of molecular ions. ${ }^{8}$ The removal of molecular interferences is critical for some masses, for example, ${ }^{30} \mathrm{SiH}$ or ${ }^{28} \mathrm{Si}_{2}$ from the analysis of ${ }^{31} \mathrm{P}$ or ${ }^{56} \mathrm{Fe}$, respectively. For semiconductor analysis by mass spectrometry, ${ }^{56} \mathrm{Fe}$ will occur at the same mass settings as ${ }^{28} \mathrm{Si}_{2}$. This is a rather significant drawback to secondary ion mass spectrometry (SIMS) analysis, since silicon material performance has been linked to low concentrations of iron, as well as other metallic impurities. ${ }^{9}$ With molecular ion interferences removed, unambiguous impurity analysis can be obtained to parts per trillion (ppt) atomic concentrations.

In addition to the removal of molecular interferences, it is also necessary to remove any possible signal that may originate from surfaces other than the sample being analyzed. In a stainless steel beamline, these surfaces are sources of $\mathrm{Fe}, \mathrm{Ni}, \mathrm{Cr}$, and other elements. Thus, reduction or elimination of such elements in the AMS source will allow high sensitivity detection of these (and other) impurities in the analysis of semiconductor materials.

This paper concentrates on the construction and fabrication of the optical components used in the ion source, previously named the Chimera source. ${ }^{10}$ These components are einzel lenses, octupole steerers, sample holder and extraction plate, Faraday cups, and aperture plates.

\section{EXPERIMENTAL APPARATUS}

\section{A. System overview}

The primary ion beam of the AMS system for semiconductor analysis is a cesium ion beam, mass selected by a $90^{\circ}$ magnetic deflection to remove impurities from the $\mathrm{Cs}^{+}$ source, and focused onto a semiconductor sample biased at a negative potential. The primary beam can be rastered across the sample for depth profiling. Negatively charged secondary ions, both atomic and molecular, formed upon ${ }^{133} \mathrm{Cs}^{+}$impact and charge exchange are extracted from the sample, analyzed for energy/charge and momentum/charge, and injected into a $3 \mathrm{MV}$ tandem accelerator. (A mass scan before injecting the negative ions into the accelerator provides a SIMS analysis.) As they pass through the accelerator terminal, the injected negative ions undergo collisions with nitrogen gas and are stripped to various positive charge states. Critical to AMS, molecular negative ions, which are the major source of uncertainty in SIMS, are dissociated. The accelerated atomic ions are then momentum/charge and energy/charge analyzed and detected in a silicon surface barrier detector, which al- 
lows energy discrimination of the ions. The limits of detection are determined in large part by the suppression of contaminant secondary ions, which can be created in the source from stray ions incident on standard vacuum hardware and ion optical elements. In order to enhance the limits of detection into the ppt range, great care must be taken to ensure that neither the incident cesium ion beam nor the extracted negative ion beam is allowed to strike any steel hardware in the beamline sections, especially in the sample chamber. In the present paper, novel optical components fabricated from crystalline silicon will be described and their operation analyzed. Through the use of these components, the analysis of semiconductor materials is being extended to sensitivities not attainable by other existing techniques.

\section{B. Optical and beamline components}

The optical components described in this paper are (1) the symmetric lenses used for focusing control of the incident and extracted beams; (2) the octupole lenses, which are operated as $x-y$ steerers of the beams; (3) a dual octupole lens operated as a raster/scanner for depth profiling; and (4) the sample chamber configuration of several components for beam shaping and the separation of the incident and extracted ion beams. Other beamline components, such as Faraday cups and collimators, are also constructed from high purity Si. The apertures of Si wafers were laser cut using a $\mathrm{CO}_{2}$ laser. All of the optical and beamline components used in the AMS source are mounted on hardened steel rails, which ensure precise alignment of the incident and extracted beam axes.

\section{CONSTRUCTION}

\section{A. Einzel lenses}

Several considerations were important for construction of the lenses used on the negative ion source. The overriding concern was that the ion beams "see" only silicon. Second, the active cylinder had to be electrically isolated up to $30 \mathrm{kV}$. Symmetric einzel lenses were chosen due to the need to control spherical aberrations over long working distance. ${ }^{11} \mathrm{~A}$ constructed lens is shown in Fig. 1. The front aperture prevents sputtering of the lens aperture with time, and can be more easily replaced than any of the lens pieces. The $1 / 2$ in. o.d. alumina balls used as electrical standoffs were set in place by dimpling both the lens cylinder and the support rods with ball-nosed end mills. In addition, stainless steel set screws in the support rods could be tightened down on the alumina balls when the lens cylinder was centered (done with the use of a customized alignment jig). The support rods were secured to the stainless steel mounting supports onto which the silicon apertures were mounted. These supports were then mounted on the steel alignment rods.

The real technical achievement that allowed the construction of the all-silicon lenses was the active lens cylinder. First, a stainless steel rod was bored out to just smaller than the o.d. of a solid, ground silicon rod. Next, the steel cylinder was heated to cause it to expand enough to allow the silicon rod to be inserted. Upon cooling, the steel cylinder contracted and firmly held the silicon rod. In this way, the silicon

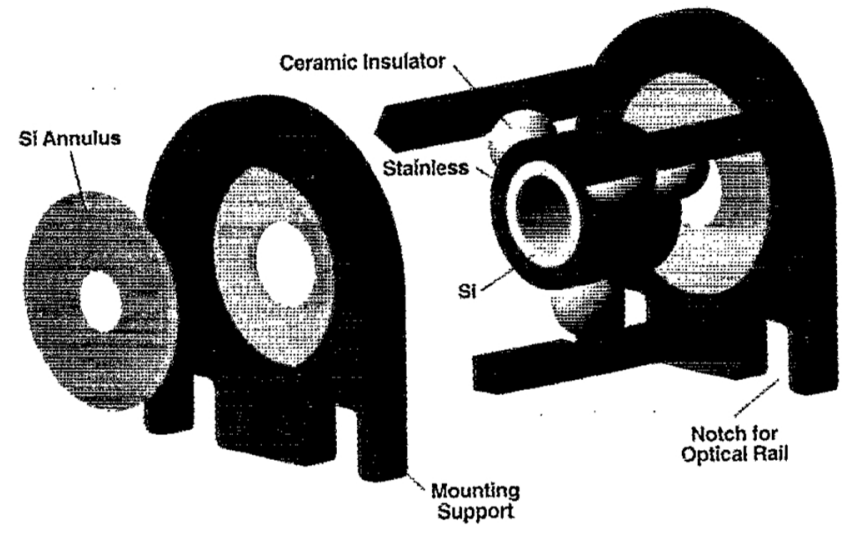

FIG. 1. A semiexploded view of an einzel lens used on the AMS negative ion source. The inner surface of the active cylinder and the front and back aperture plates of the lens are crystalline silicon. This application of silicon prevents any steel surface from being exposed to either the incident $\mathrm{Cs}^{+}$ beam or the extracted negative ion beams. The alumina balls are set into dimples in the steel sleeve of the active cylinder and provide stability as well as insulation. The voltage is applied to the lens by connecting a spring between a high voltage feedthrough and a post (not shown) on the outer steel of the inner cylinder.

could then be ground to the desired inner diameter without the shattering that normally occurs when machining silicon. Small cracks that did occur in the silicon did not change the smoothness of the inner surface nor affect the performance of the lens. The Si-in-stainless cylinder is clearly visible in Fig. 1.

Several einzel lenses are used on the negative ion source: two in the primary $\mathrm{Cs}^{+}$beamline, one lens immediately before the sample; two in the extraction line before the source electrostatic analyzer (ESA); and two in the drift tube after the ESA and before entering the existing tandem accelerator system. ${ }^{1}$ The final einzel lens of the primary line, the object lens, was made with a larger inner diameter of the active lens cylinder than the other einzel lenses. This allowed for slight rastering of the incident beam across the sample surface while keeping the beam close to the paraxial field lines of the lens.

\section{B. Octupole steerers}

The next components fabricated that provided an allsilicon appearance to the beam were electrostatic $x-y$ beam steerers. Considerations for the design of a steerer were versatility to provide dipole, quadrupole, and octupole moments simply by changing voltage configurations. One possibility was to use eight cylindrical silicon rods mounted around the beam axis. ${ }^{12}$ However, it was deemed too costly and difficult to machine smooth silicon rods, especially in comparison to laser-cutting Si wafers. A second design, which utilized the relatively inexpensive and readily available $\mathrm{Si}$ wafers, was chosen for construction.

The technical logistics of constructing the octupoles were, again, associated with electrical isolation and blade mounting. Both could be accomplished from the experience of the lens designs. Steel rods of rectangular cross section were cut to the length of silicon blades (blade thickness, 2 $\mathrm{mm}$ ). The ends were dimpled (two at one end to prevent 


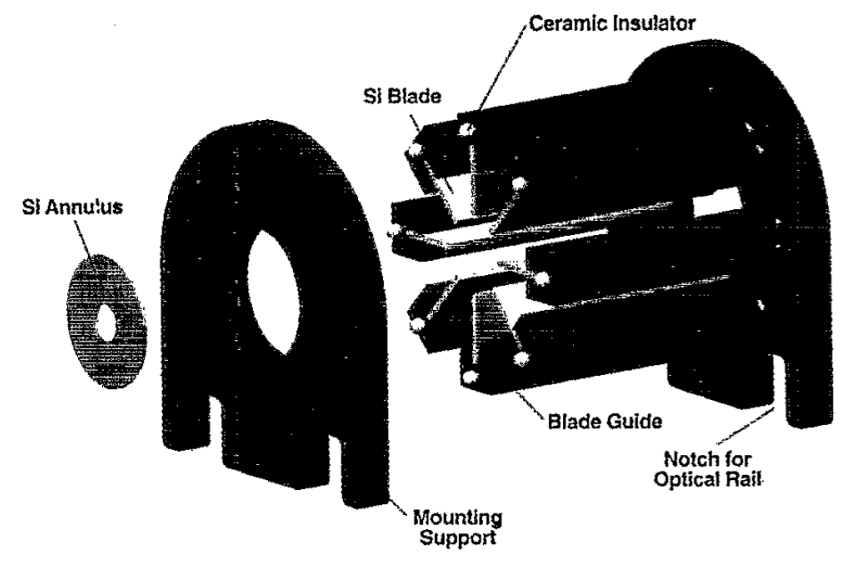

FIG. 2. A semi-exploded view of an eight-blade steerer is shown. The $1 / 8$ in. ceramic balls are explicitly shown. Ceramic balls at the far ends of the steel support rods are mounted in pairs to prevent twisting of the rods and blades during operation. Threaded rods between the mounting supports (not shown) securely hold the assembly together. Voltages are applied to the configuration by spot-welding polyimide-coated wires to the outside face of each blade guide and connecting to an eight-pin feedthrough.

rotation) with ball-nosed end mills for $1 / 8$ in. diam alumina balls, and a slot was milled the length of the steel rods for the silicon blades. The silicon blades, sandwiched between thin steel shims, were held in place by set screws from the side of the slot. The octupole design was mounted on the steel end pieces fitted with apertured silicon wafers. Looking through the apertures of the steerers, only the tips of the silicon blades can be seen. An illustration of one of these octupole steerers is shown in Fig. 2.

Through the use of a gradient field calculation software package, SIMION ${ }^{13}$ it was found that the octupole of thin, radially mounted blades would provide very parallel fields in the center of the configuration under specific voltage conditions, as desired. A plot of the blades, with respective voltages and field lines associated with horizontal steering, is shown in Fig. 3.

As with the lenses, an alignment jig was made for easier assembly of the steerers. In addition to several steerers used individually in the ion source, a doublet of equal length steerers was assembled to give "dog-leg" movement of the beam (that is, translation of the beam while keeping the direction of the beam unchanged).

A doublet consisting of two steerers of different lengths was also constructed and used for rastering the beam across the sample surface. A software program was written and downloaded to the customized power supply, which drives the rastering component. The software drives the raster in such a way that the $\mathrm{Cs}^{+}$beam is scanned across the sample surface along perimeters of ever-decreasing rectangles (with variable speed and step-size parameters), which is used for depth-profiling analysis of samples. The beam then traces itself back along the same path during which time analysis is performed. An area up to $4 \times 4 \mathrm{~mm}^{2}$ can be rastered with the hardware and software described.

\section{Sample chamber configuration}

The most critical consideration for performing contaminant-free semiconductor analysis is the positioning

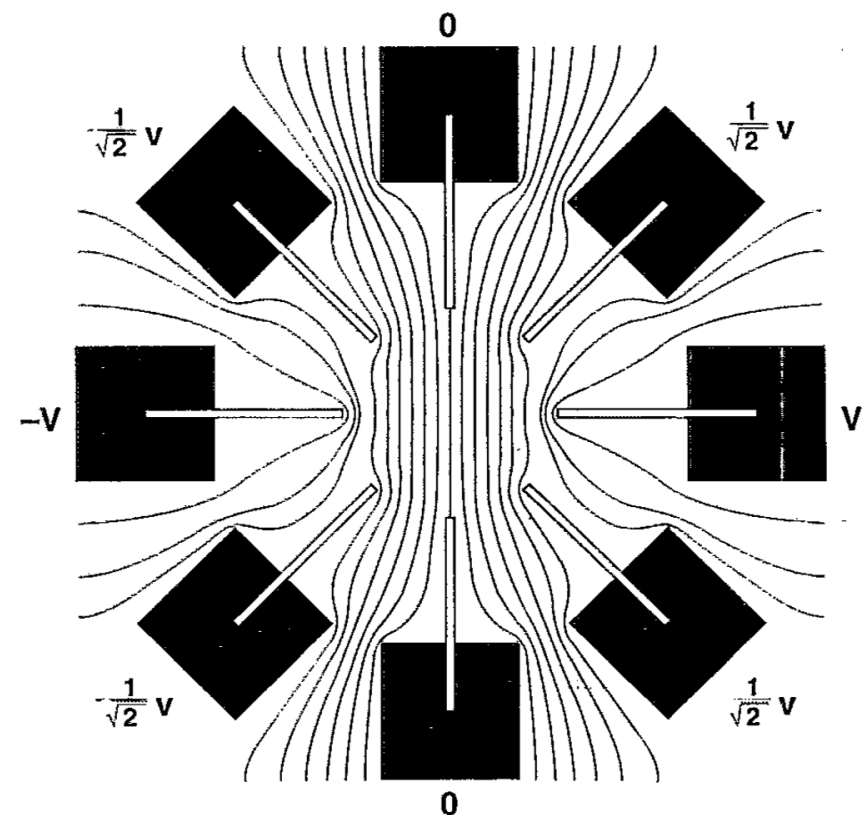

FIG. 3. SIMION equipotential plot for an eight-blade steerer. The field lines shown are for $x$ deflection of the ion beam. The field lines are seen to be parallel in the center of the blade configuration, implying uniform deflection of the beam, even when the beam is not paraxial.

of the final einzel lens in the primary beamline, the sample holder, and the initial einzel lens in the extraction beamline. The coordination of these components is necessary to extract secondary ions, which originate only from the sample surface.

The first component in the sample chamber is the object lens of the primary line, discussed above. The object lens is able to focus the primary $\mathrm{Cs}^{+}$beam down to submillimeter diameters in bulk analysis mode with achievable sizes of $<100 \mu \mathrm{m}$ diam in the depth profiling mode. With the active cylinder of this lens larger than the other einzel lenses (see Sec. III A), only $1 / 4$ in. diam alumina balls could be used for electrical isolation. However, this is still quite sufficient for isolation of the maximum $10 \mathrm{kV}$ bias voltage of the lens. For bulk mass analysis, the object lens is operated well below this voltage. The position of the object lens extends into the target chamber several centimeters. Its midpoint is $8.5 \mathrm{~cm}$ from the chamber center. The arrangement of the object lens, extraction lens, sample holder, and extraction plate, along the SIMION trajectories of the incident and extracted ion beams, is shown in Fig. 4.

The sample holder is mounted on an $x-y-z-\Theta-\phi$ manipulator. Alumina balls are again used to allow the sample to be biased at $-30 \mathrm{kV}$ with respect to ground. This bias gives a final impact energy of up to $40 \mathrm{keV}$ for the primary beam and an energy of up to $30 \mathrm{keV}$ for the extracted negative ion beam. The field caused by the sample holder bias deflects the incident beam from its $45^{\circ}$ angle of incidence to a more normal impact angle depending on the energy of the incident $\mathrm{Cs}^{+}$beam and bias voltage. For a $-30 \mathrm{kV}$ bias, the incident $\mathrm{Cs}^{+}$beam (impact energy of $40 \mathrm{keV}$ for a $10 \mathrm{keV}$ $\mathrm{Cs}^{+}$beam) is calculated to impact the sample surface roughly $20^{\circ}$ from the surface normal. For our geometry, this impact spot is more than $10 \mathrm{~mm}$ from the spot that would be 


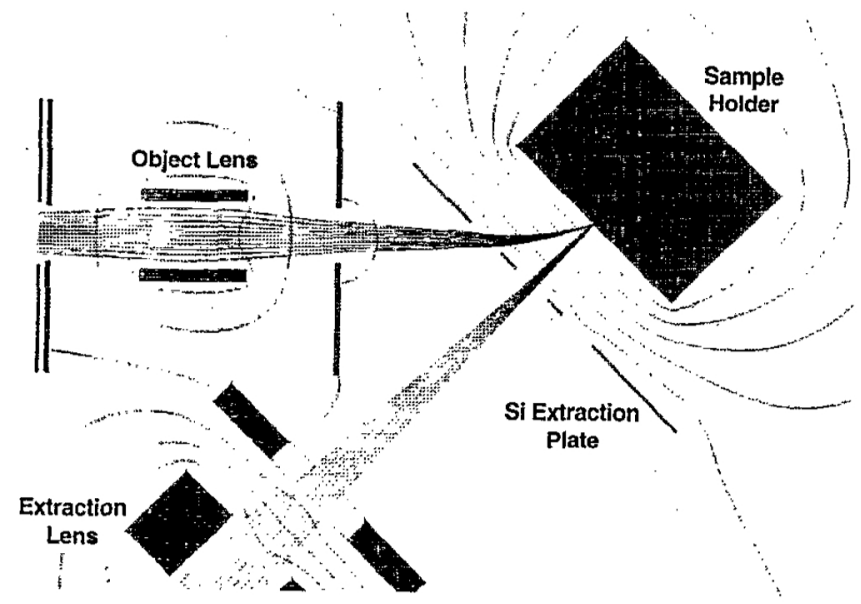

FIG. 4. Several of the optical components; object lens of the primary line, extraction lens, extraction plate, and sample holder; as well as the simION generation of the interaction of the incident $\mathrm{Cs}^{+}$beam and extracted negative ion beam in the sample chamber is shown. The deflection of the incident heam due to the sample bias is evident. The grounded Si extraction plate terminates the field lines generated by the sample bias and provides initial focusing of the extracted negative ion beam. Viewing of the sample via the long-focusing microscope is done through the unused aperture in the extraction plate.

hit by a beam following a straight line trajectory.

Up to 19 samples can be loaded simultaneously into the sample holder behind a silicon sample cover plate. The samples are spring loaded against the cover plate to ensure that the extraction optics are identical for each sample, as shown in Fig. 5(b). Even though the sample holder base is $5.7 \mathrm{~cm}$ o.d., the silicon cover plate is $8.9 \mathrm{~cm}$ o.d., to ensure that the outer samples do not experience potential field aberrations due to the edge of the sample plate. Figure 5(a) shows the front view of the sample holder.

The extraction plate is simply a grounded silicon plate with laser-cut apertures that allow entry of the primary beam, exit of the extracted negative ion beam, and viewing of the sample from a side port (see Fig. 4). The purpose of the extraction plate is to terminate the field lines generated by the sample holder voltage, provide better focusing of the extracted beam before entering the first extraction einzel lens, and shield the metal surfaces of the sample chamber from the emitted particles from the sample.

Optical viewing of the sample is obtained by mounting a $10 \times$ long-focusing microscope vertically above a halfsilvered mirror angled at $45^{\circ}$ with respect to the optical viewing port. A focused light source then illuminates the sample under analysis. The sputter crater caused by the sub-mm diameter $\mathrm{Cs}^{+}$beam is easily observed with this configuration.

\section{Faraday cups and suppressors}

As with the other components of the AMS source, the Faraday cups and suppressors are machined from crystalline silicon, with no metal parts exposed to the beam. The cups are $2.5 \mathrm{~cm}$ o.d. silicon rods cut to $3.2 \mathrm{~cm}$ lengths and turned out to form conical cups. The suppressors are silicon plates held into an aluminum frame and are held at $-100 \mathrm{~V}$. Electrical isolation of the Faraday cup is obtained by securing a
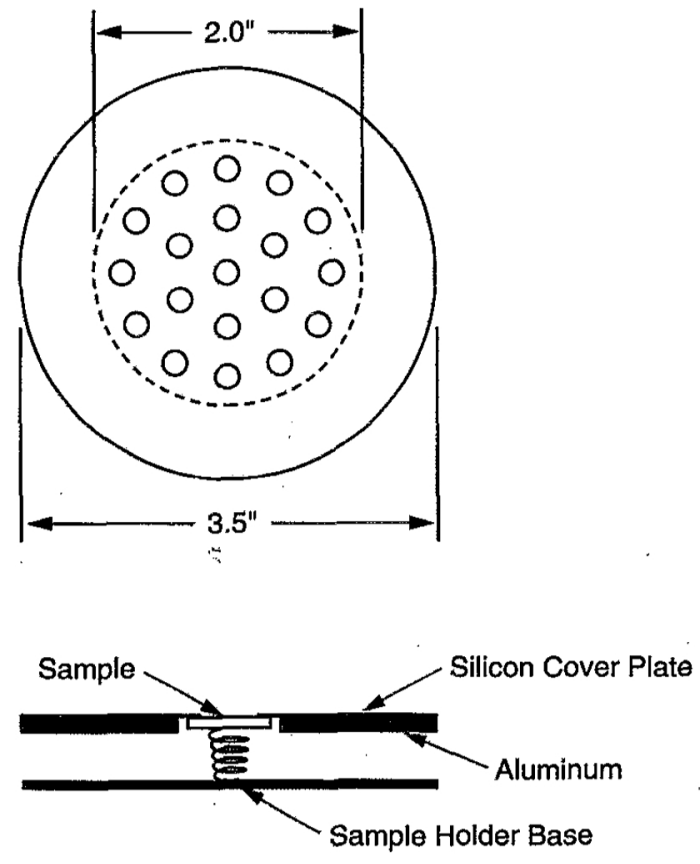

FIG. 5. (a) The front view of the sample holder shows the width of the sample cover plate with respect to the samples. The broad cover plate ensures that the field lines generated by the sample bias are equivalent for all samples without field aberrations due to the sample holder edge. Excluding samples, the surface from this view is crystalline Si. (b) A close-up view of the placement of a sample in the holder held against the Si cover plate by spring tension. The sample-to-extraction plate distance is the same for each sample, regardless of thickness. The Si cover plate is attached to the aluminum by high vacuum epoxy. Various sample holders can be made to fit the size of samples to be analyzed.

" $\mathrm{C}$ "-shaped piece of $\mathrm{Al}$ to the electropneumatic feedthrough and fitting the silicon cup inside the Al with $1 / 8$ in. alumina balls between the two. The alumina balls are set into holes in the $\mathrm{Al}$ and the Si cup is firmly held in place by the tension of the " $\mathrm{C}$ " shape of the aluminum ring and friction between the alumina balls and silicon cup.

\section{DISCUSSION}

An accelerator mass spectrometry facility is in operation at the University of North Texas' Ion Beam Modification and Analysis Laboratory for the mass analysis of semiconductor samples. An ultraclean negative ion source, which is critical to the goals of the project, has been constructed for these high-sensitivity measurements. A negative ion beam, free from beamline contaminants, is extracted from semiconductor samples after, first, mass selecting the incident $\mathrm{Cs}^{+}$beam (with a $90^{\circ}$ magnetic deflection) and, second, using novel optical components made from high purity crystalline silicon. By making all the components seen by the ion beams from pure $\mathrm{Si}$, the introduction of elements sputtered from metal surfaces is avoided and background levels are suppressed to allow detection of one part per $10^{12}$ atoms.

The components constructed are einzel lenses, octupole elements used as steerers and raster/scanners, grounded aperture plates, and Faraday cups and suppressors. Through clever construction techniques, neither the incident $\mathrm{Cs}^{+}$nor the extracted negative ion beam is allowed to impact any steel surfaces. 


\section{ACKNOWLEDGMENTS}

The authors are grateful to Larry Norton for assistance in graphics preparation and to Raksha Jain for work in preparing SIMION trajectories. This work was supported in part by the National Science Foundation Grant Nos. DMR-8812331 and ECD-9003099, the Office of Naval Research Grant Nos. N00014-89-J-1309, N00014-89-J-1344, N00014-90-J-1691, and N00014-91-J-1785, Texas Instruments Inc., Texas Utilities Electric, Inc., International Digital Modeling Corp., North Texas Research Institute, and the Robert A. Welch Foundation.

'J. L. Duggan, F. D. McDaniel, S. Matteson, D. E. Golden, J. M. Anthony, B. Gnade, and J. A. Keenan, Nucl. Instrum. Methods B 40/41, 709 (1989). 'J. M. Anthony and D. J. Donahue, Nucl. Instrum. Methods B 29, 77 (1987).

${ }^{3}$ J. M. Anthony, S. Matteson, F. D. McDaniel, and J. L. Duggan, Nucl. Instrum. Methods B 40/41; 731 (1989).

${ }^{4}$ J. C. Rucklidge, G. C. Wilson, and L. R. Kilius, Proceedings of the Fifth International Conference on Accelerator Mass Spectrometry, Paris,
France, 23-27 April 1990, edited by F. Yiou and G. M. Raisbeck (NorthHolland, Amsterdam, 1990).

${ }^{5}$ H. E. Gove, P. W. Kubik, P. Sharma, S. Datar, U. Fehn, T. Z. Hossain, J. Koffer, J. P. Lavine, S.-T. Lee, and D. Elmore, in Ref. 4.

${ }^{6}$ F. D. McDaniel, S. Matteson, J. M. Anthony, D. L. Weathers, J. L. Duggan, D. K. Marble, I. Hassan, Z. Y. Zhao, A. M. Arrale, and Y. D. Kim, J. Radiat. Nucl. Chem. 167, 423 (1993).

${ }^{7}$ R. Muller, Science 196, 489 (1977).

${ }^{8}$ K. H. Purser, R. B. Liebert, A. E. Litherland, R. P. Beukens, H. E. Gove, C. L. Bennett, M. R. Clover, and W. E. Sondheim, Rev. Phys. Appl. 12, 1487 (1977).

${ }^{9}$ J. R. Davis, Jr., A. Rohatgi, R. H. Hopkins, P. D. Blais, P. Rai-Choudhury, J. R. McCormick, and H. C. Mollenkopf, Trans. IEEE Electron. Devices ED-27, 677 (1980).

${ }^{10}$ S. E. Matteson, J. L. Duggan, D. K. Marble, F. D. McDanie1, D. L. Weathers, D. K. Wilson, J. M. Anthony, and R. L. Beavers, Nucl. Instrum. Methods B 52/53, 237 (1990).

${ }^{11}$ M. Szilaygi, Electron And Ion Optics (Plenum, New York, 1988).

${ }^{12}$ S. Matteson, D. K. Wilson, D. L. Weathers, F. D. McDaniel, and J. L. Duggan, Nucl. Instrum. Methods B 56/57, 1091 (1991).

${ }^{13}$ D. A. Dahl, J. E. Delmore, and A. D. Appelans, Rev. Sci. Instrum. 61, 607 (1990). 